\title{
D-Aspartate, a Key Element for the Improvement of Sperm Quality
}

\author{
Gemma D’Aniello ${ }^{1}$, Salvatore Ronsini ${ }^{1}$, Tiziana Notari ${ }^{1}$, Natascia Grieco ${ }^{1}$, Vincenzo Infante ${ }^{2}$, \\ Nicola D’Angelo ${ }^{2}$, Fara Mascia ${ }^{3}$, Maria Maddalena Di Fiore, George Fisher ${ }^{5}$, Antimo D’Aniello ${ }^{1,2,4, *}$ \\ ${ }^{1}$ Department of Obstetrics, Gynaecology and Physiology of Human Reproductive (IVF Unit), \\ Hospital "S. Luca", Vallo della Lucania (SA), Italy \\ ${ }^{2}$ Department of Human Clinical Chemistry of Poly-Diagnostic Center "Igea Sant'Antimo", \\ Via degli Oleandri Sant'Antimo (NA), Italy \\ ${ }^{3}$ Department of Clinical Chemistry, Diagnostic Center "Fleming Laboratory", Brescia, Italy \\ ${ }^{4}$ Department of Life Science, University of Naples Federico II, Naples, Italy \\ ${ }^{5}$ Department of Physical Sciences, Barry University, Miami Schores, USA \\ Email: daniello.antimo@gmail.com
}

Received July 18, 2012; revised August 22, 2012; accepted September 2, 2012

\begin{abstract}
Introduction: D-Aspartate is an endogenous amino acid involved in LH and testosterone release in humans. In this study we investigate the impact of nutritional supplementation of sodium D-aspartate on the improvement of sperm quality in sub-fertile patients and the rate of pregnancies that occurred with their partners. Materials and Methods: A group of 30 patients affected by oligo-asthenozoospermia and a group of 30 patients affected by asthenozoospermia were treated with a daily dose of sodium D-aspartate for 90 days. After which, the change in spermatozoa concentration and their motility and the pregnancies that occurred with their partners were recorded. Results: We found that the supplementation of D-aspartate significantly increased the concentration and the motility of spermatozoa. In oligoasthenozoospermic patients the increase of sperm concentration was found to be 2.0-fold, $\mathrm{P}<0.001$ (from a mean of $8.2 \pm$ 4.5 million spermatozoa $/ \mathrm{ml}$ of seminal plasma before treatment to a mean of $16.5 \pm 5.5$ million after treatment). In asthenozoospermic patients, the increase of spermatozoa was 1.6-fold, $\mathrm{P}<0.001$ (from a mean of $29.9 \pm 5.7$ million spermatozoa/ml before treatment to a mean of $48.7 \pm 12.8$ after treatment). The same positive effects also occurred for sperm motility. Oligo-asthenozoospermic patients showed an increase of rapid progressive spermatozoa motility from a mean of $15.5 \% \pm 4.4 \%$ before treatment to a mean of $23.1 \% \pm 4.7 \%$ after D-aspartate treatment (1.49-fold increased, $\mathrm{P}<$ 0.001). The same effects occurred in oligo-asthenozoospermic patients. In these subjects the increase of rapid progressive spermatozoa motility was 1.86 -fold (from $11.6 \% \pm 3.9 \%$ before treatment to $21.6 \pm 7.5$ after treatment, $\mathrm{P}<$ 0.001). In addition, the treatment of D-aspartate in these patients consequently led to a significantly increased number of pregnancies occurring in the partners of the treated patients. Conclusions: Treatment of sub-fertile patients with sodium D-aspartate improved the number and the motility of the spermatozoa and consequently improved the rate of pregnancies of their partners.
\end{abstract}

Keywords: D-Aspartate; Oligo-Asthenozoospermia; Asthenozoospermia; Pregnancies

\section{Introduction}

Reproductive disturbance has been a growing problem in developed countries during the last decades. According to WHO more than $10 \%$ of men worldwide are infertile, contributing to the growing demand for assisted reproduction techniques such as in vitro fertilization (IVF) and also intracytoplasmic sperm injection (ICSI) in which the quality of the spermatozoa should be the best possible option. These observations suggest that male infertility is a serious and increasing phenomenon. Various factors

*Corresponding author. are responsible for man infertility. Sociological factors, over-production of reactive oxygen species (ROS) [1], spermatozoa DNA degradation [2]. The reduction of sperm concentration and motility constitutes the main cause of male infertility. There are several literature reports of studies carried out on the use of nutritional supplements in order to improve semen quality in infertile subjects. A wide number of antioxidants have been proposed to improve sperm quality and prevent sperm damage: vitamin E, vitamin C, coenzyme Q10, folic acid, glutathione, selenium and zinc are among the molecules tested for such purposes [3]. Coenzyme Q10 plays a 
general role in transporting electrons in the mitochondria and it protects the cell membrane from oxidative stress [4]. Folic acid is involved in providing carbon group for purine and pyrimidine production and plays an important role in the synthesis of DNA [4], as well as being a potent scavenger of oxidizing free radicals by binding the methyl donors methionine and S-adenosylmethionine [6]. Lycopene and vitamins $\mathrm{A}, \mathrm{C}$ and $\mathrm{E}$ are other antioxidant reagents also used as supplementation to improve sperm quality, but no evidence exists that the use of these supplements alone can improve semen parameters [7]. Additional evidence shows that vitamin E significantly decreased DNA fragmentation rates and increased sperm quality [8]. Zinc and selenium are trace elements present in the seminal plasma that have an important antioxidant role [9]. L-carnitine and acethyl-L-carnitine [10], as well as L-Arginine [11], also have been proposed as nutritional supplements to improve sperm quality.

In this study we describe the use of another amino acid, D-aspartic acid (D-Asp), for the improvement of sperm quality in sub-fertile patients. This amino acid is an endogenous molecule occurring in nervous and endocrine glands in different species of animals including mammals and man and is involved in the hormone release at the hypothalamus hyphophysis-gonadal axis $[12,13]$. D-Asp is the stereochemical enantiomer of naturally occurring L-aspartic acid, differing by the configuration of the $\alpha$-carbon atom (Figure 1). For this reason D-Asp is metabolized specifically by the enzyme D-aspartate oxidase (EC 1.4.3.1) which oxidizes D-aspartate to oxaloacetate [14]. D-Asp is present in all endocrine glands including the testis. It also occurs in the seminal plasma and in spermatozoa, and a direct correlation between its concentration in seminal plasma and semen quality has been found [15]. In addition, D-Asp is able to stimulate testosterone synthesis in rat Leydig cells [16] and capable of improving semen quality in rabbits [17]. Lastly, we found that oral assumption of sodium D-aspartate in humans induces serum elevation of $\mathrm{LH}$ and testosterone [18]. Based on these results, in the present study we investigated whether supplementation of sodium Daspartate could improve sperm quality in oligoasthenozoospermic and asthenozoospermic subjects and consequently improve the pregnancy rate in their partners.

\section{Materials and Methods}

This study was carried out at the IVF (In Vitro Fertilization) Unit of the Department of Obstetrics, Gynecology and Physiopathology of Human Reproduction, Hospital "S. Luca", Vallo della Lucania, Italy. The study cosisted in testing the effects of sodium D-aspartate on sperm quality in oligo-asthenozoospermic and asthenzospermic patients who were unable in the least two years to impregnate their partners. These subjects came to the hospital

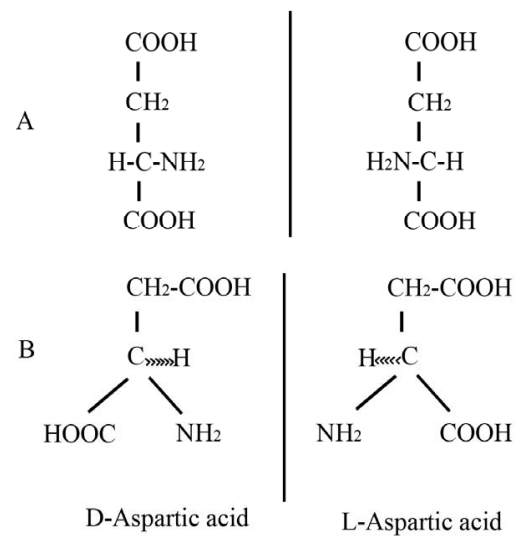

Figure 1. Linear and Stereochemical formula of D-aspartic and L-aspartic acid. Chemical structure of D-aspartic acid and L-aspartic acid by using a linear formula (panel $\mathrm{A}$ ) and a carbonium presentation based on the tetrahedral configuration (panel B).

for medical examination of semen quality and, in the case of oligoasthenozoospermia or asthenozoospermia, were prescribed to consume for 90 days an oral food supplement consisting of sodium D-aspartate, dissolved in half a glass of water or fruit juice, at lunch or at dinner. Before start experimentation, permission from all ptients was asked and obtained to conduct this study. The dose of sodium D-aspartate consisted of $10 \mathrm{ml}$ of $2.0 \mathrm{M}$ sdium D-aspartate (composed of $2.66 \mathrm{~g}$ of D-aspartic acid neutralized with $0.46 \mathrm{~g}$ of $\mathrm{NaOH}$ in $10 \mathrm{ml}$ distilled water, at final $\mathrm{pH}(6.5$ - 7.0). This food integrator was marketed in Italy by two pharmaceutical companies: 1) Pharmaguida $s r l$, which marketed the food integrator containing Daspartate under the commercial name "DADAVIT" and 2) Merck-Serono s.a.s. which marketed the food integrator containing D-aspartate under the name of "GENADIS". These companies were authorized to commercialize the integrator by the Italian Ministry of Health. The recruited subjects for this study were subdivided into two subgroups according to their concentration of spermatozoa in the seminal plasma measured before starting the treatment. The first subgroup consisted of 30 subjects affected by oligo-asthenozoospermia (ages between 27 and 43 years) who had a spermatozoa concentration ranging from $2.5 \times 10^{6}$ to $1.95 \times 10^{7}$ spermatozoa $/ \mathrm{ml}$ and a rapid progressive spermatozoa motility between $7 \%$ and $16 \%$. The second group consisted of 30 subjects affected by asthenozoospermia (ages between 28 and 40 years) who had a spermatozoa concentration raging from $2.0 \times 10^{7}$ to $5.02 \times 10^{7}$ spermatozoa $/ \mathrm{ml}$ and rapid progressive spermatozoa motility between $7 \%$ and $12 \%$.

\subsection{Semen Analyses}

Sperm ejaculates were collected by masturbation under hygienic conditions, into sterile containers, in a private room near the laboratory. Before and after D-aspartate 
treatment, semen of each patient was collected after a period of 3 days of sexual abstinence. Semen collectedbefore starting the treatment was considered as control. After semen liquefaction at $37^{\circ} \mathrm{C}$, the ejaculate volume was measured by weighing the seminal plasma. Then, a portion of $0.5 \mathrm{ml}$ was stored at $-80^{\circ} \mathrm{C}$ for future analysis of D-aspartate content, while the remaining portion of semen was used for the determination of spermatozoa concentration and motility. Sperm concentration was measured with a $100 \mu \mathrm{m}$ deep haemocytometer Neubauer chamber maintained at $37^{\circ} \mathrm{C}$. For this purpose, $0.5 \mathrm{ml}$ of seminal fluid was mixed with $0.05 \mathrm{ml}$ of a solution consisting of $20 \%(\mathrm{v} / \mathrm{v})$ formalin in $10 \%$ sodium bicarbonate $\left(\mathrm{NaHCO}_{3}\right)$ in order to block the movement of spermatozoa, to have a complete mucolisis of the semen and a homogeneous distribution of the sperm in the chamber. At least 200 spermatozoa were counted for each sperm analysis. If the seminal plasma contained a high concentration of spermatozoa, the sperm was diluted with a solution prepared by dissolution of $50 \mathrm{~g}$ of $\mathrm{NaHCO}_{3}$ and $10 \mathrm{ml}$ of formalin $35 \%(\mathrm{v} / \mathrm{v})$ in $1000 \mathrm{ml}$ of distilled water. Sperm motility was examined by using the Computer-Assistant Semen Analysis (CASA) which has the advantages of the quantitative assessment of sperm kinematics. The motility was evaluated as: 1 rapid progressive motility (spermatozoa moving actively, either linearly or in a large circles with a speed of $>25$ $\mu \mathrm{m} / \mathrm{sec}$ at $37^{\circ} \mathrm{C}$ ), 2) moderate motility (absence of progression, swimming in a small circles) and 3) slow sperm motility (slow linear movements, zigzag moving spermatozoa and vibrating in situ moving spermatozoa). Each semen analysis was carried out at the same time by two biologists. If the counts of the spermatozoa and/or the percentage of sperm motility of the two operators were not consistent, the analysis was repeated. In order to give an exact comparative evaluation of spermatozoa concentration per $\mathrm{ml}$ of ejaculate, a correction factor based on the difference of volume of the ejaculate before and after treatment was used. When the semen contained moderate to large agglutination of spermatozoa, it was not included in the study. Morphology and vitality were determined according to the guidelines of WHO manual [19].

\subsection{Determination of $D$-Aspartic Acid in Seminal Plasma}

D-Aspartic acid in the seminal plasma was determined by an HPLC method combined with the use of D-aspartate oxidase (D-AspO) as previously described [12]. The HPLC analysis (Figure 2) was carried out at the Zoological Station “A. Dohrn" of Naples, Italy and also in the laboratory of chemistry of Prof. G. Fisher at Barry University of Miami (FL) and the mean values were calculated by the comparison the two data. In brief, $0.1 \mathrm{ml}$ of seminal plasma was mixed with $0.4 \mathrm{ml}$ of $0.2 \mathrm{M}$ trichloroacetic acid and centrifuged. The supernatant was neutralized with $1.0 \mathrm{M} \mathrm{NaOH}$ and $50 \mu \mathrm{l}$ of this sample was used for the HPLC analysis.

\subsection{Serum Clinical Analysis}

In order to exclude possible side effects of sodium Daspartate treatment, each participant underwent a medical examination and various blood analyses before and after therapy to evaluate their general state of health. The blood parameter analyses were the following: 1) complete blood and platelets count (performed with HBxL80, HORIBA Medical, 34184Montpellier, France); 2) serum concentration of the following metabolites: glucose, urea, ammonia, cholesterol, triglycerides, creatinine, uric acid, sodium, potassium, calcium, magnesium, iron and copper; 3) the following enzymes: aspartate aminotransferase, alanine aminotransferase, alkaline phosphatase, acid phosphatase, prostatic phosphatase, cholinesterase and lactate dehydrogenase (performed with LaB Taurus, Instrument Laboratory SpA, Milan, Italy); 4) the following serum hormones: testosterone, luteinizing hormone, follicle stimulating hormone (FSH), thyroid stimulating hormone (TSH), triiodothyronine $\left(\mathrm{T}_{3}\right)$, thyroxin $\left(\mathrm{T}_{4}\right)$, free triiodothyronine $\left(\mathrm{FT}_{3}\right)$ and free thyroxin $\left(\mathrm{FT}_{4}\right)$ (performed with Unicell $^{\mathrm{TM}}$ Dx1800, Beckman Counter with, Inc., 2505 Kraemer BLVD, Germany); and e) serum protein electrophoresis (performed with INTERLAB G26, Interlab srl, Roma, Italy).

\subsection{Statistical Analyses}

Statistic analyses were conducted using Stat-View program (version 4, Abacus Concepts, Berkeley, CA, USA). The comparison of spermatozoon concentration $/ \mathrm{ml}$ of seminal plasma, the total sperm concentration per ejaculate and the sperm motility between the groups of subjects before and after the treatment with D-aspartate, and the concentration of D-aspartate in the seminal plasma in the subjects between before and after treatment with D-aspartate were analyzed by analysis of variance with repeated measurements (one way ANOVA). Each parameter value was reported as mean \pm SD. Statistical significance was set at $\mathrm{P}<0.05$.

\section{Results}

The results obtained in this study indicated that in both oligo-asthenozoospermic and asthenozoospermic subjects that treatment with sodium D-aspartate improved significantly the concentration of spermatozoa and their motility. Concerning the sperm concentration, we found that in oligo-asthenozoospermic patients the mean number of spermatozoa went from a basal value of $8.2 \pm 4.5$ million $/ \mathrm{ml}$ of seminal plasma to $16.5 \pm 5.5 \mathrm{million} / \mathrm{ml}$, 


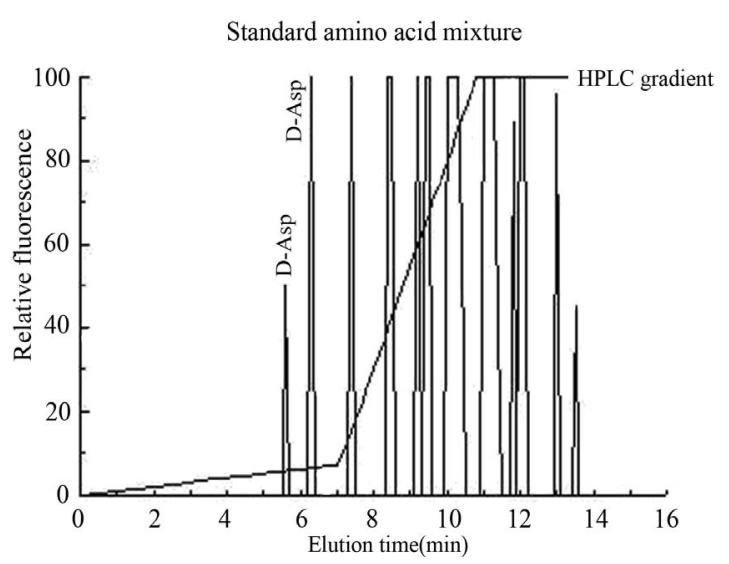

(a)

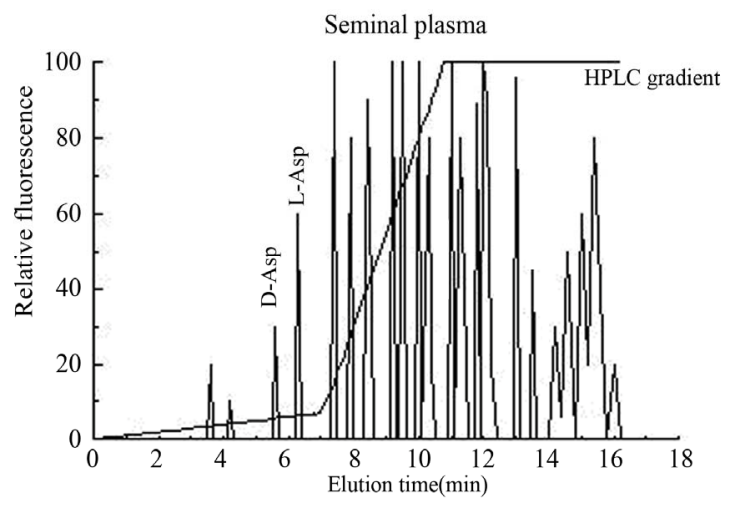

(c)

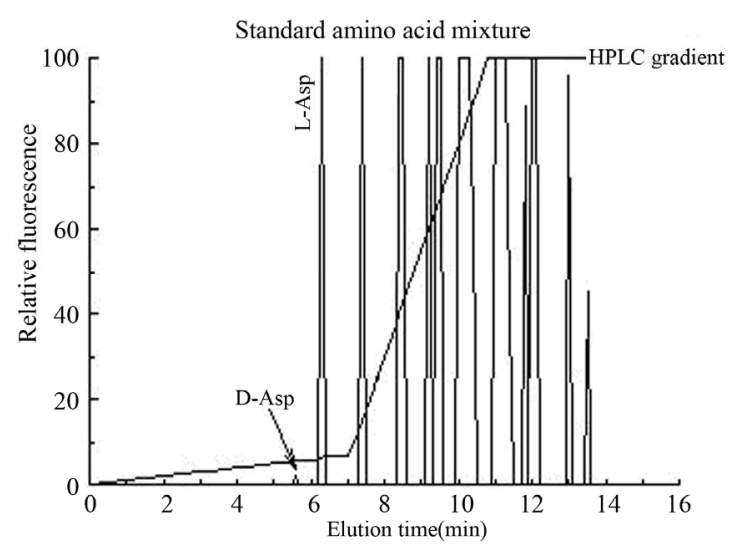

(b)

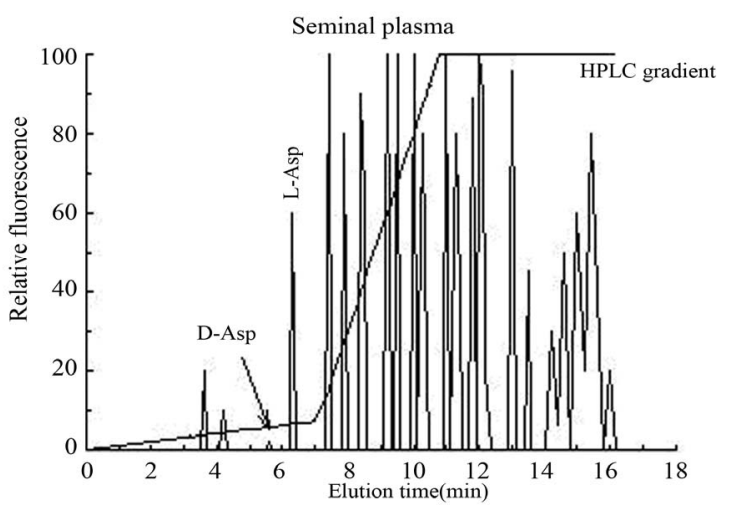

(d)

Figure 2. Typical HPLC separation and determination of D-aspartic acid from human seminal plasma. Panel (a): HPLC amino acids profile from a standard mixture consisting of 50 pmoles of D-Asp (D-aspartic acid) and 100 pmoles of L-Asp (L-aspartic acid) and 100 pmoles of other standard L-amino acids; Panel (b): HPLC profile of the same amino acids standard mixture as used in panel (a), but after treatment with $D$-aspartate oxidase (D-AspO) to oxidize D-Asp in order to ascertain if the peak observed at elution time of $5.6 \mathrm{~min}$, was actually D-Asp; Panel (c): HPLC analysis of free amino acids of typical seminal plasma from a normospermic patient purified on cation exchange resin corresponding to $10 \mu \mathrm{l}$ of the original sample; Panel (d): HPLC analysis of the same sample as used in panel (c), but after treatment with D-AspO. The peak of D-Asp completely disappeared from the standard mixture of amino acids (panel (b)) and from the seminal plasma (panel (d)), after incubation of the sample with $D$-aspartate oxidase, indicating that the peak area of D-Asp was entirely due to D-Asp.

showing a 2.0 -fold increase $(\mathrm{P}<0.001)$. The same effect was observed on the total concentration of spermatozoa per ejaculate that went from $30.1 \pm 14.7$ million/ejaculate to $45.9 \pm 24.4$ million/ejaculate, a 1.52 -fold increase $(\mathrm{P}<$ 0.001) (Table 1). An analogous improved phenomenon was observed in the asthenozoospermic patient group, where a significant increase of the number of spermatozoa occurred, going from a basal value of $29.9 \pm 5.7$ mil$\mathrm{lion} / \mathrm{ml}$ of seminal fluid to $48.7 \pm 12.6 \mathrm{million} / \mathrm{ml}$, with a 1.63 -fold increase $(\mathrm{P}<0.001)$. The total number of spermatozoa/ejaculate was also significantly increased by 1.57-fold, (from a value of $97.1 \pm 36.1 \mathrm{million} /$ ejaculate to $153.0 \pm 47.0$ million/ejaculate $(\mathrm{P}<0.001)$ (Table 1). Concerning the spermatozoa motility, we found a significant increase of spermatozoa motility in both oligoasthenozoospermic and asthenozoospermic patients subjects. In the group of oligo-asthenozoospermic subjects, the mean value of the rapid progressive sper Nmatozoa motility went from $15.5 \% \pm 4.4 \%$ to $23.1 \% \pm 4.7 \%(1.49$ fold increase, $\mathrm{P}<0.001)$, and the total sperm motility also increased significantly from $30.6 \% \pm 9.3 \%$ to $54.4 \%$ $\pm 9.7 \%$ (1.77-fold increase, $\mathrm{P}<0.001)$ (Table 2). In the group of asthenozoospermic subjects, sodium D-aspartate treatment also induced a significant increase of rapid progressive motility and of total spermatozoa motility. In this case, in fact the treatment induced a 1.86 -fold increase in rapid progressive motility $(\mathrm{P}<0.001)$ going from a basal value of $11.6 \% \pm 3.9 \%$ before treatment to $21.6 \% \pm 7.5 \%$ after treatment, and the total sperm motility went from $25.2 \% \pm 8.1 \%$ to $45.7 \% \pm 10.9 \%(1.81$ fold increased) (Table 2).

An interesting aspect of this study is the very significant increased number of pregnancies that occurred in the partners of the treated patients. In fact, among the 60 partners of subjects who were treated with sodium Daspartate, 16 of them became pregnant which corresponds 
Table 1. Sperm concentration in oligo-asthenozoospermic and asthenozoospermic patients treated with sodium Daspartate.

\begin{tabular}{|c|c|c|c|}
\hline \multicolumn{4}{|c|}{ 1st group: 30-Oligo-Asthenozoospermic patients } \\
\hline \multicolumn{2}{|c|}{$\begin{array}{l}\text { Concentration of spermatozoa/ } \\
\text { ml of seminal plasma } \\
\left(\mathrm{n} . \times 10^{6}\right)\end{array}$} & \multicolumn{2}{|c|}{$\begin{array}{l}\text { Total concentration of } \\
\text { spermatozoa/ejaculate }\left(\mathrm{n} . \times 10^{6}\right)\end{array}$} \\
\hline Before & After & Before & After \\
\hline Treatment & Treatment & Treatment & Treatment \\
\hline $8.2 \pm 4.5$ & $16.5 \pm 5.5$ & $30.1 \pm 14.7$ & $45.9 \pm 24.4$ \\
\hline \multicolumn{2}{|c|}{$\mathrm{P}<0.001$} & \multicolumn{2}{|c|}{$\mathrm{P}<0.001$} \\
\hline \multicolumn{4}{|c|}{ 2nd group: 30-Asthenozoospermic patients } \\
\hline \multicolumn{2}{|c|}{$\begin{array}{l}\text { Concentration of spermatozoa/ } \\
\mathrm{ml} \text { of seminal plasma }\left(\mathrm{n} . \times 10^{6}\right)\end{array}$} & \multicolumn{2}{|c|}{$\begin{array}{c}\text { Total concentration of } \\
\text { spermatozoa/ejaculate }\left(n . \times 10^{6}\right)\end{array}$} \\
\hline Before & After & Before & After \\
\hline Treatment & Treatment & Treatment & Treatment \\
\hline $29.9 \pm 5.7$ & $48.7 \pm 12.6$ & $97.1 \pm 36.1$ & $153.0 \pm 47.0$ \\
\hline \multicolumn{2}{|c|}{$\mathrm{P}<0.001$} & \multicolumn{2}{|c|}{$\mathrm{P}<0.001$} \\
\hline
\end{tabular}

* Refers to the total number of spermatozoa obtained by multiplying the spermatozoa concentration $/ \mathrm{ml}$ of ejaculate by total volume of the ejaculate. The mean and SD were obtained by one-way ANOVA with repeated measurements (Stat-View program). The $\mathrm{P}$ value was obtained by comparison of the data of each group of subjects between before and after D-aspartate treatment.

Table 2. Sperm motility in oligo-asthenozoospermic and asthenozoospermic patients treated with sodium D-aspartate.

\begin{tabular}{|c|c|c|c|}
\hline \multicolumn{4}{|c|}{ 1st group: 30-Oligo-Asthenozoospermic patients } \\
\hline \multicolumn{2}{|c|}{$\begin{array}{l}\text { Rapid progressive }{ }^{*} \text { spermatozoa } \\
\text { motility ( } \% \text { of total) }\end{array}$} & \multicolumn{2}{|c|}{$\begin{array}{c}\text { Total spermatozoa }{ }^{* *} \text { motility } \\
\text { ( } \% \text { of total) }\end{array}$} \\
\hline Before & After & Before & After \\
\hline Treatment & Treatment & Treatment & Treatment \\
\hline $15.5 \pm 4.4$ & $23.1 \pm 4.7$ & $30.6 \pm 9.3$ & $54.4 \pm 9.7$ \\
\hline \multicolumn{2}{|c|}{$\mathrm{P}<0.001$} & \multicolumn{2}{|c|}{$\mathrm{P}<0.001$} \\
\hline \multicolumn{4}{|c|}{ 2nd group: 30 -Asthenozoospermic patients } \\
\hline \multicolumn{2}{|c|}{$\begin{array}{l}\text { Rapid progressive }{ }^{*} \text { spermatozoa } \\
\text { motility ( } \% \text { of total) }\end{array}$} & \multicolumn{2}{|c|}{$\begin{array}{c}\text { Total spermatozoa }{ }^{* *} \text { motility } \\
\text { ( } \% \text { of total) }\end{array}$} \\
\hline Before & After & Before & After \\
\hline Treatment & Treatment & Treatment & Treatment \\
\hline $11.6 \pm 3.9$ & $21.6 \pm 7.5$ & $25.2 \pm 8.1$ & $45.7 \pm 10.9$ \\
\hline \multicolumn{2}{|c|}{$\mathrm{P}<0.001$} & \multicolumn{2}{|c|}{$\mathrm{P}<0.001$} \\
\hline
\end{tabular}

The patients in this study were the same patients reported in table 1. "Rapid progressive spermatozoa motility indicated those spermatozoa with a forward motility $>25 \mu \mathrm{m} / \mathrm{s}$ measured at $37^{\circ} \mathrm{C}$, expressed as the percent of rapid progressive spermatozoa motility on total spermatozoa. ${ }^{* *}$ Total spermatozoa motility indicate the sum of rapid progressive moving spermatozoa, the slow progressive moving spermatozoa, the zigzag moving spermatozoa and the vibrating in situ moving spermatozoa and are expressed as total motile spermatozoa on the total. The mean and SD were obtained by one-way ANOVA with repeated measurements (Stat-View program). The $\mathrm{P}$ value was obtained by comparison of the data of each group $f$ patients between before and after D-aspartate.

to a pregnancy rate of $26.6 \%$. In detail, of six pregnancies that occurred among the 30 partners of the oligoasthenozoospermic patients two of them became pregnant at the second month that their partners were treated with
D-aspartate, and four of them became pregnant at the third month that their partners were treated with Daspartate. In contrast, of 10 pregnancies among 30 partners of the asthenozoospermic patients, two of them became pregnant after one month of treatment, four of them became pregnant after two months of treatment, and four of them became pregnant after three months of treatment (Table 3).

The last interesting aspect of this study regards the concentrations of D-Asp in the seminal fluid before and after treatment. We measured the concentration of D-Asp in seminal plasma of all patients before and after oral consumption of sodium D-aspartate at the dose as aboveindicated (see: Materials and Methods). The results indicated that this amino acid increases significantly in concentration following treatment with D-Asp. In fact, themean concentration of D-Asp in oligoasthenzoo-spermic patients before treatment was $15.0 \pm 4.0 \mathrm{nmol} / \mathrm{ml}$ of seminal plasma, whereas after treatment it increased to $29.5 \pm 7.2 \mathrm{nmol} / \mathrm{ml}(1.96$-fold increased, $\mathrm{P}<0.001)$. In asthenozoospermic patients before treatment $\mathrm{D}$-aspartate concentration was $16.3 \pm 4.5 \mathrm{nmol} / \mathrm{ml}$ of seminal plasma, whereas after treatment it increased to $32.6 \pm 7.5 \mathrm{nmol} / \mathrm{ml}$ (2.0-fold increased, $\mathrm{P}<0.001)$ (Table 4).

In order to verify if $\mathrm{D}$-aspartate treatment could induce unwanted side effects, each patient that had been treated with D-aspartate underwent a full blood clinical analysis (as previously detailed in the methods section). None of the blood metabolites as well as the complete blood and platelets count was outside the physiological range. The only variation was observed in the $\mathrm{LH}$ and testosterone

Table 3. Number of pregnancies occurring to the partners of oligo-asthenozoospermic patients and asthenozoospermic patients before and after treatment with sodium D-aspartate.

\begin{tabular}{|c|c|c|c|c|}
\hline \multicolumn{5}{|c|}{$\begin{array}{l}\text { Numbers of pregnancies occurring to the partners of } \\
\text { the } 30 \text { oligo-asthenozoospermic patients }\end{array}$} \\
\hline $\begin{array}{c}\text { Before } \\
\text { D-aspartate }\end{array}$ & \multicolumn{4}{|c|}{ After D-Aspartate treatment } \\
\hline \multirow[t]{2}{*}{ Treatment $^{*}$} & After & After & After & $\%$ of total ${ }^{*}$ \\
\hline & 1 month & 2 months & 3 months & \\
\hline $0 \%$ & $0 \%$ & $2 \%$ & $4 \%$ & $20 \%$ \\
\hline \multicolumn{5}{|c|}{$\begin{array}{l}\text { Numbers of pregnancies occurring to the partners } \\
\text { of the } 30 \text { asthenozoospermic patients }\end{array}$} \\
\hline \multirow{3}{*}{$\begin{array}{c}\text { Before } \\
\text { D-aspartate } \\
\text { Treatment }^{*}\end{array}$} & \multicolumn{4}{|c|}{ After D-Aspartate treatment } \\
\hline & After & After & After & $\%$ of total ${ }^{*}$ \\
\hline & 1 month & 2 months & 3 months & \\
\hline $0 \%$ & $2 \%$ & $4 \%$ & $33 \%$ & $20 \%$ \\
\hline
\end{tabular}

*Before treatment indicate the number of pregnancies occurred during the last two years before starting the D-aspartate treatment. After treatment indicate the number of pregnancies occurred to the partners of subjects after the first, the second and the third month of an daily oral dose of D-aspartate. 
Table 4. D-Aspartic acid concentration in seminal plasma of patients before and after treatment with sodium D-Aspartate (nmole/ml).

\begin{tabular}{|c|c|c|}
\hline & $\begin{array}{c}\text { Before } \\
\text { treatment }\end{array}$ & $\begin{array}{c}\text { After } \\
\text { treatment }\end{array}$ \\
\hline \multicolumn{3}{|l|}{ 1st group } \\
\hline \multicolumn{3}{|c|}{30 Oligo-Asthenozoospermic patients } \\
\hline Mean $\pm \mathrm{SD}$ & $15.0 \pm 4.0$ & $29.5 \pm 7.2$ \\
\hline Student's t-test & \multicolumn{2}{|c|}{$\mathrm{P}<0.001$} \\
\hline \multicolumn{3}{|l|}{ 2nd group: } \\
\hline \multicolumn{3}{|c|}{30 Asthenozoospermic patient } \\
\hline Mean $\pm \mathrm{SD}$ & $16.3 \pm 4.5$ & $32.6 \pm 7.5$ \\
\hline Student's t-test & \multicolumn{2}{|c|}{$\mathrm{P}<0.001$} \\
\hline
\end{tabular}

The determination of D-aspartic acid was carried out by HPLC by using the OPA-NAC method [19]. The statistical data (mean, SD and P value) were obtained by using the one-way ANOVA, with repeated measurements (StatView program).

concentrations that were found to be increased between 1.3 - 1.6 fold compared to their basal levels in the Daspartate group. However, the increased levels of LH and testosterone observed in this study were in agreement with the previously reported results [18] demonstrating that D-aspartate has the capacity to increase LH and testosterone blood levels. Therefore, D-aspartate treatment for a prolonged time (90 days) is in no way harmful to health.

\section{Discussion}

D-Aspartic acid is an endogenous amino acid in animals and is synthesized in the endocrine glands, principally in the testis, by the conversion of L-aspartate to D-aspartate by a D-aspartate racemase [18]. Previous studies have demonstrated that D-aspartic acid is involved in endocrine activity in animals and humans in the synthesis and release of sexual hormones [12,16-18,20-24]. Also it has been ddemonstrated that D-aspartate occurs in human seminal plasma and spermatozoa and that a direct relationship between semen quality and D-aspartate concentration exists [18]. These data thus led us to hypothesize that D-aspartate plays an important role in steroidogenesis and that its supplementation in sub-fertile patients (oligo-asthenozoospermic and asthenozoospermic) could improve spermatozoa concentration and motility and consequently the rate of pregnancies of their partners. Therefore, we enrolled 60 patients, who in the previous two years have not fertilized their partners, and treated them for three months with an oral supplementation of sodium D-aspartate. The results obtained from this study have demonstrated that $\mathrm{D}$-aspartate induces a significant increase of the number of spermatozoa and of their motility in both oligo-asthenozoospermic and in asthenozoospermic patients, and this phenomenon in turn has resulted in a very significant increase of the pregnancies in the women partners of subjects treated with D-aspartate. In fact, among the 30 partners of the oligoasthenozoospermic patients treated with D-aspartate, six of them became pregnant (rate $=20 \%$ ), and among the 30 partners of the asthenozoospermic subjects treated with Daspartate 10 became pregnant which corresponds to $33 \%$ of total. In this regard, two pregnancies already occurred one month after their partners had ingested D-aspartate, and four pregnancies occurred after the second month that their partners were treated with D-aspartate. The last interesting result obtained in this study is the fact that the consumption of D-aspartate for a time of almost three months did not have any side effects on the health of the subjects. In fact, we have monitored the principal serum metabolites indicated for the usual clinic analyses in all subjects before and after D-aspartate treatment, and we have observed that in each patient treated with D-aspartate all serum parameters were within the physiological values, indicating thus that $\mathrm{D}$-aspartate treatment do not cause any damages to the health.

In conclusion, in this study we demonstrated that sub-fertile men who consumed daily an oral dose of sodium D-aspartate for 2 - 3 months, improved signifycantly their sperm quality in terms of number and mobility of the spermatozoa, and consequently they increased the possibility to fertilize the own partner. We do not know the exact molecular mechanism by which this phenomenon occurs, but we strongly hypothesize that this events is due to action of D-aspartate in inducing elevation of testosterone in the testis, which locally is involved in improving the spermatogenesis and in maturation of male gametes.

\section{Acknowledgements}

The authors thank Dr. Antimo Cesaro, Administrator of the "Polydiagnostic Center "Igea Sant'Antimo", 80029 Sant'Antimo (Italy), Dr. Giuseppe Cammisa, Director of the Chemical Clinical Laboratory of the same Center and the technicians Nicola Migliaccio and Gianfranco Silvestre for their support in clinical analysis. We also thank the Zoological Station “A. Dohrn" of Naples, Italy, for the permission to carried out HPLC analysis. A special thanks to Dr. Fara Mascia of the "Fleming Laboratory" of Brescia (Italy) for her observations and discussion about sperm analyses.

\section{REFERENCES}

[1] A. Agarwal, R. A. Saleh and M. A. Bedaiwy, "Role of Reactive Oxygen Species in the Pathophysiology of Human Reproduction" Fertility Sterility, Vol. 79, No. 4, 2003, pp. 161-164. doi:10.1016/S0015-0282(02)04948-8

[2] E. T. Donnelly, M. O'Connell, N. McClure and S. E. Lewis, "Differences in Nuclear DNA Fragmentation and 
Mitochondrial Integrity of Semen and Prepared Human Spermatozoa," Human Reproduction, Vol. 15, No. 7, 2000, pp. 1552-1561. doi:10.1093/humrep/15.7.1552

[3] A. Agarwal and L. H. Sekhon, "The Role of Antioxidant Therapy in the Treatment of Male Infertility," Human Fertility, Vol. 3, No. 4, 2010, pp. 217-225. doi:10.3109/14647273.2010.532279

[4] M. Bentinger, M. Tekle and G Dallner, "Coenzyme QBiosynthesis and Functions," Biochem Biophys Research Communication, Vol. 396, No. 1, 2010, pp. 74-79. doi:10.1016/j.bbrc.2010.02.147

[5] I. M. Ebisch, G.M. Thomas, W. H. Peters, D. D. Braat and R. P. Steegers-Theunissen, "The Importance of Folate, Zinc and Antioxidants in the Pathogenesis and Prevention of Subfertility," Human Reproductive Update, Vol. 13, No. 2, 2007, pp. 163-174. doi:10.1093/humupd/dml054

[6] R. Joshi, S. Adhikari, B.S. Patro, S. Chattopadhyay and T. Mukherjee, "Free Radical Scavenging Behavior of Folic Acid: Evidence for Possible Antioxidant Activity," Free Radical Biological Medicine, Vol. 30, No. 12, 2010, pp. 1390-1399. doi:10.1016/S0891-5849(01)00543-3

[7] C. Rolf, T. G. Cooper, C. H. Yeung and E. Nieschlag, "Antioxidant Treatment of Patients with Asthenozoospermia or Moderate Oligoasthenozoospermia with HighDose Vitamin C and vitamin E: A Randomized, PlaceboControlled, Double-Blind Study," Human Reproduction, Vol. 14, No. 4, 2009, pp. 1028-1033. doi:10.1093/humrep/14.4.1028

[8] E. Greco, M. Iacobelli, L. Rienzi, F. Ubaldi, S. Ferrero, and J. Tesarik, "Reduction of the Incidence of Sperm DNA Fragmentation by Oral Antioxidant Treatment," Journal of Andrology, Vol. 26, No. 3, 2005, pp. 349-353.

[9] C. Boitani and R. Puglisi, "Selenium, a Key Element in Spermatogenesis and Male Fertility," Advances Experimental Medicine Biology, Vol. 636, 2008, pp. 65-73. doi:10.1007/978-0-387-09597-4_4

[10] A. Lenzi, P. Sgrò, P., Salacone, D. Paoli, B Gilio, et al., "A Placebo-Controlled Double Blind Randomized Trial of the Use of Combined L-Carnitine and L-Acetyl-carnitine Treatment in Men with Asthenozoospermia," Fertility Sterility, Vol. 81, No. 6, 2004, pp.1578-1584. doi:10.1016/j.fertnstert.2003.10.034

[11] J. P. Pryor, J. P. Blandy, P. Evans, D. M. Chaput De Saintonge and M. Usherwood, "Controlled Clinical Trial of Arginine for Infertile Men with Oligozoospermia," British Journal of Urology, Vol. 50, No. 1, 1978, pp. 4750. doi:10.1111/j.1464-410X.1978.tb02765.X

[12] A. D’Aniello, M. M. Di Fiore, G. H. Fisher, A. Milone, A. Seleni, et al., "Occurrence of D-Aspartic Acid and N-Methyl-D-aspartic Acid in Rat Neuroendocrine Tissues and Their Role in the Modulation of Luteinizing Hormone and Growth Hormone Release," The FASEB Journal, Vol. 14, No. 5, 2000, pp. 699-714.

[13] A. D'Aniello, "D-Aspartic Acid: An Endogenous Amino Acid with an Important Neuroendocrine Role," Brain Research Reviews, Vol. 53, No. 2, 2007, pp. 215-234. doi:10.1016/j.brainresrev.2006.08.005

[14] A. D'Aniello, A. Vetere and L. Petrucelli, "Further Study on the Specificity of D-Amino Acid Oxidase and D-Aspartate Oxidase and Time Course for Complete Oxidation of D-Amino Acids," Comparative Biochemistry Physiology B, Vol. 105, No. 2, 1993, pp. 731-734. doi:10.1016/0305-0491(93)90113-J

[15] G. D'Aniello, S. Ronsini, F. Guida, P. Spinelli and A. D'Aniello, "Occurrence of D-Aspartic Acid in Human Seminal Plasma and Spermatozoa: Possible Role in Reproduction," Fertility and Sterility, Vol. 84, No. 5, 2005, pp. 1444-1449. doi:10.1016/j.fertnstert.2005.05.019

[16] Y. Nagata, H. Homma, J. A. Lee and K. Imai, "D-Aspartate Stimulation of Testosterone Synthesis in Rat Leydig Cells," FEBS Letters, Vol. 444, No. 2-3. 1999, pp. 160164. doi:10.1016/S0014-5793(99)00045-9

[17] G. Macchia, E. Topo, N. Mangano, E. D’Aniello and R. Boni, "D,L-Aspartic Acid Administration Improves Semen Quality in Rabbit Bucks," Animal Reproductive Science, Vol. 118, No. 2-4, 2010, pp. 337-343. doi:10.1016/i.anireprosci.2009.07.009

[18] E. Topo, A. Soricelli, A. D'Aniello, S. Ronsini and G. D'Aniello, "The Role and Molecular Mechanism of DAspartic Acid in the Release and Synthesis of LH and Testosterone in Humans and Rats," Reproductive Biology Endocrinology, Vol. 7, No. 1, 2009, pp. 120-131. doi:10.1186/1477-7827-7-120

[19] World Health Organization, "WHO Laboratory Manual for the Examination and Processing of Human Semen," 50th Edition, Cambridge University Press, New York, 2010.

[20] K. Imai, T. Fukushima, K. Hagiwara and T. Santa, "Occurrence of D-aspartic Acid in Rat Brain Pineal Gland," Biomedicine Chromatography, Vol. 9, No. 2, 1995, pp. 106-109. doi:10.1002/bmc.1130090211

[21] A. D’Aniello, A. Di Cosmo, C. Di Cristo, L. Annunziato, L. Petrucelli, et al., "Involvement of D-aspartic Acid in the Synthesis of Testosterone in Rat Testes," Life Science, Vol. 59, No. 2, 1996, pp. 97-104. doi:10.1016/0024-3205(96)00266-4

[22] A. D'Aniello, M. M. Di Fiore, G. D'Aniello, F. E. Colin, G. Lewis, B. B. Setchell, "Secretion of D-Aspartic Acid by the Rat Testis and Its Role in Endocrinology of the Testis and Spermatogenesis," FEBS Letters, Vol. 436, No. 1, 1998, pp. 23-27. doi:10.1016/S0014-5793(98)01087-4

[23] K. Sakai, H. Homma, J. Lee, T. Fukushima, T. Santa, Tashiro, T. Iwatsubo, K. Imai. "Localization of D-Aspartic Acid in Elongate Spermatids in Rat Testis," Archives of Biochemistry and Biophysics, Vol. 351, No. 1, 1988, 96105. doi:10.1006/abbi.1997.0539

[24] M. Pampillo, T. Scimonelli, M. C. Bottin, B. H. Duvilanski and V. Rettori, "The Effect of D-Aspartate on Luteinizing Hormone-Releasing Hormone, $\alpha$-Melanocyte-stimulating Hormone, GABA and Dopamine Release," Neuroreport, Vol. 13, No. 17, 2002, pp. 2341-2344. doi:10.1097/00001756-200212030-00034 